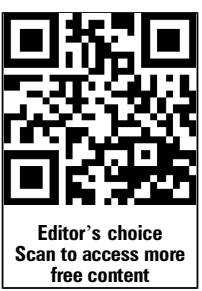

- Additional material is published online only. To view please visit the journal online (http://dx.doi.org/10.1136/ ejhpharm-2014-000483).

${ }^{1}$ Strathclyde Institute of Pharmacy and Biomedical Sciences, University of Strathclyde, Glasgow, UK 2Pharmacy Department, Western Infirmary, Glasgow, UK

${ }^{3}$ Pharmacy Department, Crosshouse Hospital, Kilmarnock, UK

${ }^{4}$ NHS National Services Scotland, Public Health Intelligence, Edinburgh, UK

\section{Correspondence to} Dr Rosemary Newham, Strathclyde Institute of Pharmacy and Biomedical Sciences, University of Strathclyde, 161 Cathedra Street, Glasgow G4 ORE, UK; rosemary.e.newham@strath.ac. uk

Received 28 April 2014 Revised 26 June 2014 Accepted 2 July 2014 Published Online First 25 July 2014

\title{
Barriers to the safe and effective use of intravenous gentamicin and vancomycin in Scottish hospitals, and strategies for quality improvement
}

\author{
R Newham, ${ }^{1}$ A H Thomson, ${ }^{1,2}$ Y Semple, ${ }^{1}$ S Dewar, ${ }^{3}$ T Steedman, ${ }^{4}$ M Bennie ${ }^{1,4}$
}

\begin{abstract}
Objectives Avoiding harm when patients interact with healthcare services is a global issue. Guidelines are often produced to improve the prescribing and monitoring of medication with a narrow therapeutic index. Adherence to guidelines may not occur in clinical practice. This paper aims to explore the barriers faced by clinical pharmacists and junior doctors when using complex guidelines to support the prescribing and therapeutic drug monitoring of gentamicin and vancomycin.

Methods Twenty-three junior doctors and 27 clinical pharmacists took part in focus groups at four hospital sites in four Scottish health boards between March and July 2011. Focus groups were run separately at each hospital site for the two clinical groups. The data were organised using the framework approach, validated, and a thematic analysis was conducted.
\end{abstract}

Results Five themes emerged to explain barriers to effective initial prescribing and therapeutic drug monitoring, which were divided into two types. Barriers could be a direct result of the content of the guidelines - specifically that clinicians required experience to use the guidelines effectively. Barriers also resulted from a failure in the context in which the guidelines function which was related to insufficient dissemination, communication issues within the hospital site, unmet educational needs and staffing issues.

Conclusions Improved patient safety cannot be assumed due to the existence of gentamicin and vancomycin guidelines. The findings of this study highlight the complexities associated with their appropriate use. Future quality improvement strategies must consider where the guidelines will be implemented, and the context in which they will function.

\section{INTRODUCTION}

Internationally, it is likely that harm is suffered by millions of patients due to their contact with healthcare services-with approximately one in ten patients in secondary care suffering an adverse event. ${ }^{1}$ In 2008, Scotland implemented a national patient safety programme, which revolutionised how patient safety was approached across a major healthcare system. The latest data suggest that a $12.4 \%$ reduction in hospital mortality rates has been recorded since the programme's inception. ${ }^{2}$ One of the key aims of the Scottish Patient Safety Programme (SPSP) was to reduce healthcareassociated infections. ${ }^{3}$ The Scottish Antimicrobial Prescribing Group (SAPG) was formed in 2008 to improve antimicrobial prescribing and management across NHS Scotland. A key focus was to reduce the use of broad spectrum antimicrobials, which risk selecting out pathogenic organisms, such as Clostridium difficile. ${ }^{4} 5$ SAPG published national guidelines recommending the use of narrow spectrum antimicrobials, such as gentamicin, as first-line therapy to treat a variety of infections. This policy change resulted in an increase in aminoglycoside use in Scottish hospitals, with a $8.1 \%$ increase over the period 2010-2011. ${ }^{6}$ To support these new antimicrobial guidelines, SAPG produced specific guidance on intravenous gentamicin and vancomycin dosage regimens and therapeutic drug monitoring (TDM).

Previous audits examining adherence to TDM guidelines for gentamicin and vancomycin ${ }^{8-13}$ have demonstrated poor compliance with recommendations. For example, only $58.7 \%$ of vancomycin use was in line with local guidance for dose, dose frequency and TDM measures in a Dutch intensive care unit, ${ }^{12}$ while $34 \%$ of initial gentamicin dosing was concordant with Australian hospital guidelines. ${ }^{9}$ No previous studies have examined barriers to the appropriate use of TDM for gentamicin and vancomycin although it has been suggested that merely providing guidelines and education is not sufficient to ensure compliance, ${ }^{9}$ and that further, multidisciplinary, research into the implementation of antimicrobial guidelines is needed. ${ }^{14}$

The present study evaluated the application of the SAPG TDM guidelines for intravenous gentamicin and vancomycin that were published in October 2009. ${ }^{7}$ These guidelines comprised a single vancomycin regimen and two gentamicin dosing regimens; health boards decided which gentamicin regimen was adopted. The guidelines included recommendations for initial dosage regimens (including an online dose calculator for vancomycin and one of the gentamicin dosage regimens) and nomograms with advice to support the interpretation of antibiotic concentration measurements. As an adjunct to the guidelines, a case scenario involving gentamicin and vancomycin was included as part of the mandatory training on the use of antimicrobials provided to junior doctors through a national online training system; pharmacist training was through undergraduate and postgraduate education. There was no national training package specifically for gentamicin or vancomycin for pharmacists. By December 2010, 80\% of NHS Scotland health boards had adopted the gentamicin and the vancomycin guidelines. ${ }^{15}$ 
A point prevalence study ${ }^{15}$ conducted in parallel with this qualitative study identified issues with adherence to the guidelines: the initial prescribed doses were consistent with recommendations in only $65 \%$ of patients prescribed vancomycin, and $61 \%$ of patients prescribed gentamicin, while the first sample taken for TDM was in accordance with guidelines in 64\% and $74 \%$ of cases, respectively. These findings supported the need for exploratory research to describe the challenges associated with ensuring the safe and effective use of gentamicin and vancomycin.

\section{METHODS}

A qualitative methodology was used, as the flexible questioning technique allows areas salient to the participants to be investigated $^{16}$ while being suited to exploring drug-prescribing decisions. ${ }^{17}$ Four Scottish NHS health boards, representative of the geographical and population spread across Scotland, were selected. All four boards had implemented the gentamicin, as well as the vancomycin national guidelines.

\section{Ethical considerations}

As the project was part of a service evaluation, NHS ethical approval was not required. ${ }^{18}$

\section{Data collection}

A standard topic guide was developed through discussions within the multidisciplinary evaluation team, which comprised pharmacists, a social scientist and an information analyst. This was piloted with a combined group of pharmacists and doctors at a hospital site not subsequently included in the final sample, and amendments were made as necessary. The key areas covered were the use of the hospital guidelines, and the challenges and improvements which could be made to the prescribing and monitoring of gentamicin and vancomycin (see online supplementary material for topic guide). The focus groups were conducted between March 2011 and July 2011.

One hospital was selected from each of the four health boards, and the final sample comprised two moderately sized (500-600 beds) and two large (over 800 beds) hospitals. From these hospitals, junior doctors and clinical pharmacists were invited to take part in focus groups. Recruitment was initially via the hospitals' antimicrobial pharmacists through standardised recruitment letters and opportunistic sampling (whereby potential participants were approached directly). Additionally, participants were asked to approach any colleagues they thought would be interested. Fifty front-line clinicians (27 pharmacists and 23 doctors) were recruited-table 1 summarises their basic demographic information presented by health board and profession.

Gentamicin and vancomycin were discussed separately in the focus groups using identical topic guides. To limit bias due to order effects (ie, that the participants' answers to the first antimicrobial could affect their responses to the second), which antimicrobial was discussed first alternated between focus groups. Additionally, to avoid hierarchical effects and group status differences, doctors' focus groups were carried out separately from pharmacists' groups. One focus group was conducted for each profession at each hospital site, with the exception of Hospital 2 where two focus groups were run with the pharmacists to increase participant numbers. $\mathrm{RN}$ moderated all focus group discussions except in Hospital 3 where a clinical pharmacist moderated the discussion around gentamicin and RN moderated the discussion around vancomycin. To aid the subsequent
Table 1 Characteristics of the pharmacists and doctors who participated in the focus groups $(n=50)$

\begin{tabular}{|c|c|c|c|}
\hline Health board & & $\begin{array}{l}\text { Clinical } \\
\text { pharmacists }\end{array}$ & $\begin{array}{l}\text { Junior } \\
\text { doctors }\end{array}$ \\
\hline $\begin{array}{l}\text { Hospital } 1 \\
\text { (500-600 beds) }\end{array}$ & $\begin{array}{l}\text { Number ( } \% \text { female) } \\
\text { Years' experience: } \\
\% \leq 1 \text { year } \\
\% 2-9 \text { years } \\
\% \geq 10 \text { years }\end{array}$ & $\begin{array}{l}8(75) \\
12.5(n=1) \\
37.5(n=3) \\
50(n=4)\end{array}$ & $\begin{array}{l}8(75) \\
87.5(n=7) \\
12.5(n=1) \\
-\end{array}$ \\
\hline $\begin{array}{l}\text { Hospital } 2 \\
\text { (over } 800 \text { beds) }\end{array}$ & $\begin{array}{l}\text { Number ( } \% \text { female) } \\
\text { Years' experience (SD) } \\
\% \leq 1 \text { year } \\
\% 2-9 \text { years } \\
\% \geq 10 \text { years }\end{array}$ & $\begin{array}{l}8(75) \\
25(n=2) \\
12.5(n=1) \\
62.5(n=5)\end{array}$ & $\begin{array}{l}5(80) \\
100(n=5) \\
- \\
-\end{array}$ \\
\hline $\begin{array}{l}\text { Hospital } 3 \\
\text { (500-600 beds) }\end{array}$ & $\begin{array}{l}\text { Number ( } \% \text { female) } \\
\text { Years' experience (SD) } \\
\% \leq 1 \text { year } \\
\text { 2-9 years } \\
\% \geq 10 \text { years }\end{array}$ & $\begin{array}{l}6(67) \\
50(n=3) \\
16.67(n=1) \\
33.33(n=2)\end{array}$ & $\begin{array}{l}4(25) \\
100(n=4) \\
- \\
-\end{array}$ \\
\hline $\begin{array}{l}\text { Hospital } 4 \\
\text { (over } 800 \text { beds) }\end{array}$ & $\begin{array}{l}\text { Number ( } \% \text { female) } \\
\text { Years' experience (SD) } \\
\% \leq 1 \text { year } \\
\% 2-9 \text { years } \\
\% \geq 10 \text { years }\end{array}$ & $\begin{array}{l}5(80) \\
- \\
20(n=1) \\
80(n=4)\end{array}$ & $\begin{array}{l}6(50) \\
50(n=3) \\
50(n=3) \\
-\end{array}$ \\
\hline Total sample & $\begin{array}{l}\text { Number ( } \% \text { female) } \\
\text { Years' experience (SD) } \\
\% \leq 1 \text { year } \\
\% 2-9 \text { years } \\
\% \geq 10 \text { years }\end{array}$ & $\begin{array}{l}27(74) \\
22.22(n=6) \\
22.22(n=6) \\
55.56(n=15)\end{array}$ & $\begin{array}{l}23(61) \\
82.6(n=19) \\
17.4(n=4)\end{array}$ \\
\hline
\end{tabular}

transcription, a second researcher attended all focus groups and noted who was speaking.

Each focus group lasted up to $90 \mathrm{~min}$, with refreshments provided. All participants read a standardised information sheet, were informed that they could withdraw from the study, were assured that they would remain anonymous, and gave their informed, written consent prior to taking part in the evaluation. The focus groups were recorded using a dictaphone and later transcribed verbatim and validated through a second researcher comparing the transcript to the audio and correcting any discrepancies. All participants were allocated a participant number, and this, in addition to the second researcher's notes, was used to ensure accuracy and anonymity of the subsequent transcription.

\section{Analysis}

The analysis focused on the general research question: how the guidelines for gentamicin and vancomycin supported front-line clinicians (pharmacists and doctors) to deliver safe and effective patient care and the barriers which prevented effective application of the guidelines.

A thematic analysis was carried out after organising the data using the framework approach, which enables analysis to be carried out on a large volume of data using a transparent methodology. ${ }^{19}{ }^{20}$ Frameworks based on the research question, the topic guide and four transcripts were created independently by two researchers. These were compared and any areas of disagreement were resolved through consensus between the researchers to create a single, validated framework into which the remaining transcripts were coded. During the subsequent analysis, emerging themes were discussed by the authors of this paper with regards to their face-validity and appropriateness in line with the quotations.

Although the technical details regarding dosing regimens and monitoring differed between the antimicrobials, the issues raised 
for gentamicin and vancomycin were generally similar and, therefore, they are described together. Furthermore, dosing guidelines and the online calculator were taken as being synonymous, as many participants reported using the online guidelines linked to the calculator, where available.

The analysis reflects general themes expressed by participants and quotes have been selected to illustrate them. On occasion, words contained in parentheses have been added to the quotes to clarify meaning or disguise the hospital site involved. Removed text is represented by ellipses. Positive utterances made by the interviewer or coparticipants to encourage the respondents, and hesitations, have been omitted to increase the overall coherence.

\section{RESULTS}

Various themes emerged to explain barriers to effective use of the TDM guidelines. These were divided into two types: those which were directly linked to the content of the guidelines ('direct barriers'); and those resulting from a failure of the environment within which the guidelines operate, and the organisational structure in place to support their use ('indirect barriers'). Quotations which reflect these barriers and themes are presented in figure 1 .

\section{Direct barriers}

Need for experience when using the TDM guidelines

It was observed by pharmacists and doctors that the guidelines did not negate the need for experience in making judgement calls, especially if the antibiotic concentration measurements came back at the cut-off point for treatment modification (Quotation 1), and a minor deviation from the expected concentration could cause a large change in the dosing regimen
(Quotation 2). This issue was compounded by a lack of understanding regarding the importance of accurate documentation of dose and sample times. It was felt that this, in association with a lack of experience, could potentially lead to an inappropriate modification of the dosing regimen (Quotation 3).

\section{Indirect barriers}

Lack of awareness of TDM guidelines

Guideline use was hindered by a lack of awareness. The importance of an effective strategy through which the guidelines were implemented was highlighted, for example, that consultants may lack awareness regarding the new guidelines which may lead to doses being prescribed that were not consistent with the recommendations (Quotation 4), or a junior doctor not using the available calculator for dosage calculations (Quotation 5). One pharmacist focus group, reflecting on their own guideline implementation process, considered that informing the consultants about the guidelines through an email may have resulted in a lack of awareness (Quotation 6).

\section{Communication issues within hospital sites}

The problems of identifying patients who were receiving either of the antimicrobials were discussed. No site had a hospitalwide process for communicating who had been prescribed vancomycin or gentamicin; however, different wards within a hospital had developed systems to ensure everyone was aware of the monitoring needs of patients on gentamicin and vancomycin (Quotation 7), while communication at the weekend, when there was limited pharmacist support, was seen to be problematic (Quotation 8). A lack of information transfer when patients moved between wards was also highlighted as a problem, as this

9. "There's no hand over if a patient moves wards as well from you know, there's no clear cut introduction to the FY1 "this patient is coming up and they're on gentamicin or vancomycin"'" (Participant 31; FY1)

1. "We had somebody who was on the line basically and it was either 24 hour or the 48 hour dosing and [the pharmacist] said, "Well, because the renal function is improving, let's just - and cause then their infections quite bad let's go with the $\mathbf{2 4}$ hours as opposed to the $\mathbf{4 8}$ hours". So I never really had to make that decision, I just kinda agreed with her." (Participant 31; FY1)

2. "So it's that grey area that's just either if you're just ever so slightly under. And sometimes I've asked people and they've said,"no just leave [the dose] up. I'm sure [the concentration] will come up" or something you know? I think a pharmacist said that to me before or the registrar or something and true enough it has. They've used it more so they know." (Participant 50; FY1)

3. "... The level was misinterpreted as the timing of it [was off] and that the difference of the few hours that it was misinterpreted caused them to halve the dose.". (Participant 10; Pharmacist band 8)

Theme 2: Lack of awareness of guidelines

4. "I do still find it odd the sort of older prescriber who is perhaps not been familiar with the therapeutics handbook or the online calculator just giving a gram bd [of vancomycin] and hoping for the best" (Participant 16; Pharmacist band 8)

5. A: Just the palaver of working out the equation when you know you want something done quickly and you have got other things to do...

$B:$ the calculator is excellent isn't it?

A: I've never used it (A: Participant 38, FY1; B: Participant 37, FY1)

6. "... the guidelines got circulated as a [general] email. This is the new policy and consultants pressed delete." (Participant 23; Pharmacist band 7)

Theme 3: Communication issues within hospital sites

7. "... on the ward I was in we [communicated in] three ways. We did it in the diary, the hand over diary, we did it on the Kardex and we also did it on ... the chart on the wall." (Participant 17; Pharmacist band 6)

8. "On a Friday I give the junior doctor that's on a list of all the current gent and vanc patients and they know to follow them up but then there's new patients, we're obviously not on the ward, the new patients they have to just work out themselves but they all know about the protocols." (Participant 23; Pharmacist band 7)

Figure 1 Themes and quotations arising from pharmacist and clinician focus groups conducted in 4 health board areas in Scotland. 
meant that new patients arriving on a ward may not be monitored or might miss a dose (Quotation 9).

\section{Unmet educational needs}

The issue of poor documentation of blood sampling times and consequent problems of interpreting results was raised by pharmacists and doctors (Quotation 10) and the importance of documenting the data entered into the online calculator was raised (Quotation 11). Additionally, the importance of obtaining an accurate weight (Quotation 12) and height (Quotation 13) was not fully appreciated by respondents, which suggested an educational need.

\section{Staffing issues}

Staffing levels were highlighted as being insufficient within most hospital sites, particularly at the weekends and out of hours. Due to low staffing levels, the prioritisation of tasks was highlighted as a problem. Monitoring was regarded as being less important than many other tasks which the foundation-level doctors were expected to perform (Quotation 14). At times, it was not clear who was responsible for each aspect of monitoring, which led to confusion between staff and differences between wards (Quotation 15). For example, in some wards, nursing staff were actively involved in the monitoring, whereas in other wards-perhaps where the antibiotics were used lessthis was not the case. While the guidelines were primarily used by foundation-year doctors and by pharmacists, promoting the guidelines to the nurses-and therefore increasing their responsibility—was suggested as means of improving patient safety (Quotation 16).

\section{DISCUSSION}

The analysis identified a number of barriers to effective use of the TDM guidelines. The themes were divided into two 'types'-barriers directly resulting from the guidelines themselves (specifically, content not providing sufficient support for inexperienced staff) and barriers resulting from the context in which the guidelines were functioning (lack of awareness of guidelines, communication issues within hospital site, unmet educational needs for effective use of the guidelines and staffing issues).

These themes support previous research which suggests that providing guidelines is not sufficient to ensure compliance. ${ }^{9}$ Although there is no equivalent research in the TDM literature, parallels can be drawn between the present findings and the general literature on guidelines. The current study proposes that inadequacies in the organisational structure were multifaceted, comprising a lack of support through inadequate staffing, communication issues and a failure to disseminate the guidelines effectively. This is concordant with environmental factors raised as impediments to the effective implementation of clinical guidelines. ${ }^{21-23}$ Our findings are also consistent with a recent qualitative study examining the antimicrobial prescribing experience of junior doctors which highlighted context as rendering antimicrobial prescribing challenging. ${ }^{24}$ However, within healthcare systems, the routine redesign of infrastructure with the release of new guidelines is unfeasible and undesirable, and guidelines must be designed to operate within the available resources. To address this, guidelines could demonstrate, prior to their adoption within a healthcare system, the path within the multidisciplinary team that successful adoption would follow.

The observation that educational and experiential needs act as a barrier to effective TDM when using complex guidelines echoes the results of Cabana et al, who found that gaps in physicians' knowledge were a barrier to following general antimicrobial guidelines. ${ }^{23}$ It is important that guidelines are seen as a 'reasonable' starting point rather than the only option. Modifications to dosage guidelines may be necessary in some clinical situations, and this requires experience.

This is the first study to explore barriers to the safe and effective use of gentamicin and vancomycin through the adoption of complex TDM guidelines. By understanding barriers, interventions can be planned with the intended user in mind to circumvent common pitfalls to the effective implementation of guidelines. $^{14}$

Behavioural change models may be used to assess and plan interventions to support effective implementation of guidelines. A taxonomy of behaviour change techniques has recently been proposed with the aim of building international consensus for reporting on interventions. ${ }^{25}$ Common to all models of behaviour change is the influence of capability, opportunity and motivation (known as the COM-B model). ${ }^{26} \mathrm{~A}$ change in behaviour may require a sustained change in one or more of the COM elements. Our study provides examples of barriers to capability (need for experience and education), opportunity (communication issues and a lack of awareness of guidelines) and motivation (competing priorities linked to staffing issues), showing the importance of theory-guided interventions to overcome potential barriers. Interventions to enhance capability include decision support, case review with reflection, and feedback of information about achievement of agreed goals. ${ }^{25}$ This is consistent with Mattick et al's suggestion of three different strategies to improve antimicrobial prescribing practice: context-specific information about practice, case discussion with senior colleagues and regular feedback about practice. ${ }^{24}$ Our results also support ensuring that the infrastructure exists to support the optimisation of gentamicin and vancomycin therapy for effective application of the guidelines. A team-based approach may alter the behaviour of clinicians by increasing the perceived importance of individualisation of therapy at an institutional level.

\section{Limitations of evaluation}

There were certain limitations associated with the present evaluation. First, the participants were self-selected. Consequently, those who were interviewed may not have been representative of typical medical and pharmacy staff in the hospital. All those interviewed were generally positive about the content of the guidelines, which may be due to a selection bias. Additionally, the issue of generalisability within qualitative research is an important one. Focus groups give insight to the issues most salient to the group interviewed-although the observation that no further themes emerged in the final focus groups suggested that saturation had been reached. Additionally, it may be that the responses given were functional to explain the failure of participants within these groups to follow the guidelines. However, the parallels between the present findings and the general antimicrobial guideline implementation literature suggest that the findings generalise beyond the present context. Additionally, this evaluation was undertaken at a national level within a devolved healthcare system covering a population of around 5.2 million with 14 geographical health boards. The homogenous nature of the population, and the sampling strategy to recruit from a range of geographical sizes and locations, increases the generalisability of the results.

\section{Implications for future practice}

Having a strong evidence base for guidelines is necessary but not sufficient to ensure their suitability for practice. The results 
of the present study-combined with those from a national point prevalence study ${ }^{15}$-informed the agenda for a national quality improvement programme (QIP) for gentamicin and vancomycin use. This has included the creation of additional national resources to support the use of the TDM guidelines; specialised documentation, targeted educational material, and an electronic calculator. However, while a national strategy will tackle some of the barriers identified within this paper, the importance of tailoring locally to meet site-specific needs must not be underestimated. ${ }^{22}$

A recent Cochrane review of the audit and feedback paradigm concluded that presenting evaluations of the application of guidelines to clinicians was an effective method for increasing compliance with a desired practice. ${ }^{27}$ This was enhanced by the specification of a specific, measurable, timed goal, and by the use of action planning when feedback shows a gap between performance and goal. ${ }^{27}$ The results of the current project were fed back to the antimicrobial teams within the hospital sites and health boards, and could provide a foundation for designing a future audit and feedback intervention to assess outcomes and continue the quality improvement cycle.

\section{CONCLUSIONS}

Complex guidelines to ensure the safe and effective use of gentamicin and vancomycin may improve patient safety, but there are barriers to their use and, therefore, guidelines alone do not provide a definitive answer. Our results highlight the importance of the infrastructure within which guidelines operate. Ensuring that an infrastructure exists to support the optimisation of gentamicin and vancomycin therapy will encourage effective application of the guidelines.

\section{Key messages}

What is already known on this subject

- There is an increasing use of narrow spectrum antibiotics (including gentamicin and vancomycin) to prevent and treat hospital-associated infections.

- Gentamicin and vancomycin require therapeutic drug monitoring (TDM) to ensure their safe and effective use.

\section{What this study adds}

- TDM guidelines should be tailored to the local organisational structure to enable effective implementation and communication across the multidisciplinary team.

- To support safe practice, TDM guidelines should reflect users' knowledge base, with additional educational needs addressed as necessary.

Acknowledgements We are grateful to Kirsten McCulloch and the antimicrobial pharmacists who aided our recruitment, and to all the doctors and pharmacists who generously gave their time to attend the focus groups. Also, our thanks go to the researchers who supported the validation and transcription of the audio recordings. The results have not been published previously in any peer-reviewed publication. The study findings have been presented orally and as a NHS Report to the funding body. The study findings have also been presented as posters. This work was conducted as part of a gentamicin and vancomycin quality improvement programme (GaV QIP) that was commissioned by the NHS Scotland HAI Task Force (Healthcare Acquired Infection), in collaboration with the Scottish Antimicrobial Prescribing Group (SAPG).

Contributors RN: Study design, data collection, qualitative analysis, discussion of results, preparation of manuscript, agreed final version of manuscript. MB, AHT and YS: Study design, discussion of results, preparation of manuscript, agreed final version of manuscript. TS and SD: Study design, discussion of results, preparation of manuscript.

Competing interests All authors have completed the Unified Competing Interest form at http://www.icmje.org/coi_disclosure.pdf (available on request from the corresponding author) and declare RN, YS and SD had part-time support from NHS Scotland HAI Task Force.

Provenance and peer review Not commissioned; externally peer reviewed.

\section{REFERENCES}

1 World Health Organization. Global priorities for patient safety research: Better knowledge for safer care. 2009. http://whqlibdoc.who.int/publications/2009/ 9789241598620_eng.pdf (accessed 28 Apr 2014).

2 Royal Pharmaceutical Society. Scottish safety programme credited with drop in mortality. Pharm J 2013;290:262

3 NHS Scotland. Scottish Patient Safety Programme. 2008. http://www.scottishpatientsafetyprogramme.scot.nhs.uk/programme/news?resid=682 (accessed 12 Dec 2013).

4 Department of Health and Health Protection Agency. Clostridium difficile infection: how to deal with the problem. 2009. http://www.dh.gov.uk/prod_consum_dh/groups/dh_ digitalassets/documents/digitalasset/dh_093218.pdf (accessed 28 Nov 2012).

5 Berrington A, Borriello SP, Brazier J, et al. National Clostridium difficile standards group: report to the department of health. J Hosp Infect 2004;56:1-38.

6 Health Protection Scotland and Information Services Division. Report on Antimicrobial Use and Resistance in Humans in 2011. http://www.isdscotland.org/ Health-Topics/Prescribing-and-Medicines/Publications/2013-02-26/2013-02-26SAPG-Report.pdf (accessed 16th December 2012).

7 Scottish Antimicrobials Prescribing Group. Scottish Medicines Consortium: Guidance on use of gentamicin and vancomycin in adults. 2009. http://www. scottishmedicines.org.uk/files/SAPG_Guidance_on_gentamicin_and_vancomycin policies_revised.pdf (accessed 22 Nov 2010).

8 Rogers MS, Cullen MM, Boxall EM, et al. Improved compliance with a gentamicin prescribing policy after introduction of a monitoring form. J Antimicrob/ Chemother 2005:56:566-8.

9 Leong $\mathrm{CL}$, Buising K, Richards $\mathrm{M}$, et al. Providing guidelines and education is not enough: an audit of gentamicin use at The Royal Melbourne Hospital. Intern Med J 2006;36:37-42.

10 Kadambari S, Heath PT, Sharland M, et al. Variation in gentamicin and vancomycin dosage and monitoring in UK neonatal units. J Antimicrob Chemother 2011;66:2647-50.

11 Swartling M, Gupta R, Dudas V, et al. Short term impact of guidelines on vancomycin dosing and therapeutic drug monitoring. Int I Clin Pharma 2012:34:282-5

12 Minne L, Eslami S, Kuiper RA, et al. Five years of therapeutic drug monitoring in the intensive care did not change vancomycin prescription behaviour: perceived needs for decision support. Minerva Anestesiol 2012;78:684-92.

13 Traugott KA, Maxwell PR, Green K, et al. Effects of therapeutic drug monitoring criteria in a computerized prescriber-order-entry system on the appropriateness of vancomycin level orders. Am J Health Syst Pharm 2011;68:347-52.

14 Charani E, Edwards R, Sevdalis N, et al. Behavior Change Strategies to influence antimicrobial prescribing in acute care: a systematic review. Clin Infect Dis 2011;53:651-62.

15 Gentamicin and Vancomycin Quality Improvement Programme Team. NHS Scotland Gentamicin and Vancomycin (GaV) Quality Improvement Programme ReportJanuary 2012

16 Ritchie J. The application of qualitative methods to social research. In: Ritchie J, Lewis J, eds. Qualitative research practice: a guide for social science students and researchers. London: Sage, 2003:24-46.

17 Stevenson FA, Britten N, Barry CA, et al. Qualitative methods and prescribing research. J Clin Pharm Ther 2000;25:317-24.

18 Department of Health. Governance arrangements for NHS Research Ethics Committees. 2001. http://www.dh.gov.uk/en/Publicationsandstatistics/Publications/ PublicationsPolicyAndGuidance/DH_4005727 (accessed 23 May 2012).

19 Pope C, Ziebland S, Mays N. Qualitative research in health care-analysing qualitative data (reprinted from qualitative research in health care). BMJ 2000:320:114-16.

20 Ritchie J, Spencer L, O'Connor W, eds. Carrying out qualitative analysis. London: Sage Publications Ltd., 2003.

21 Barlow G, Nathwani D, Myers E, et al. Identifying barriers to the rapid administration of appropriate antibiotics in community-acquired pneumonia. J Antimicrob Chemother 2008;61:442-51.

22 Majumdar SR, Simpson SH, Marrie TJ. Physician-perceived barriers to adopting a critical pathway for unity-acquired pneumonia. Jt Comm J Qual Saf 2004;30:387-95.

23 Cabana MD, Rand CS, Powe NR, et al. Why don't physicians follow clinical practice guidelines? A framework for improvement. JAMA-J Am Med Assoc 1999;282:1458-65. 
24 Mattick K, Kelly N, Rees C. A window into the lives of junior doctors: narrative interviews exploring antimicrobial prescribing experiences. J Antimicrob Chemother 2014 doi: 10.1093/jac/dku093.

25 Michie S, Richardson M, Johnston M, et al. The behavior change technique taxonomy (v1) of 93 hierarchically clustered techniques: building an international consensus for the reporting of behavior change interventions. Ann Behav Med 2013;46:81-95.

26 Michie S, van Stralen MM, West R. The behaviour change wheel: a new method for characterising and designing behaviour change interventions. Implementation Sci 2011;6:42.
27 Ivers N, Jamtvedt G, Flottorp S, et al. Audit and feedback: effects on professional practice and healthcare outcomes. Cochrane Database Syst Rev 2012(6):CD000259.

28 Semple Y, Dewar S, Thomson A, et al. National study of gentamicin and vancomycin use to inform development of a quality improvement programme. Federation of Infection Societies Conference. Birmingham, 2013.

29 Semple Y, Dewar S, Thomson A, et al. Development of a quality improvement programme to improve patient safety and clinical effectiveness of gentamicin and vancomycin. Chester: United Kingdom Clinical Pharmacy Association, 2013. 